\title{
THE ORDERABILITY AND CLOSED IMAGES OF SCATTERED SPACES
}

\author{
S. PURISCH
}

\begin{abstract}
A (totally) orderable scattered space and a space homeomorphic to a subspace of an ordinal space are characterized in terms of a neighborhood subbase for each of their points plus what corresponds to a neighborhood base for each of their non- $Q$-gaps. These generalize the characterizations in [ $\mathrm{P}_{1}$ ] of an orderable compact scattered space and in [B] of a space homeomorphic to a compact ordinal space. Generalizing a result in $[\mathrm{M}]$ it is shown that a space is orderable and scattered iff it is the 2 to 1 image under a closed map of a subspace of an ordinal space. In response to a question of Telgarsky [T] a simple description is given of a closed map with discrete fibers from an orderable scattered space onto an orderable perfect space. Maps that preserve length conditions on a scattered space are touched upon.
\end{abstract}

\section{INTRODUCTION}

A space is scattered if each of its nonempty subsets has an isolated point, and it is suborderable if it is embeddable in an orderable space.

In $\left[\mathrm{P}_{2}\right]$ it was shown that a suborderable scattered space is orderable and admits an orderable scattered compactification. Compact scattered spaces that are orderable were characterized in $\left[\mathrm{P}_{1}\right]$. Hence a scattered space is orderable iff it admits an orderable scattered compactification. But a more intrinsic characterization is desirable. The elimination here of the compactness condition in $\left[\mathrm{P}_{1}\right]$ causes problems necessitating new terminology.

In this paper a sequence $S$ is a copy of a cofinal subspace of a regular ordinal $\tau$. A limit point $p$ of $S$, where $p \notin S$, corresponds to the point $\tau$ under an embedding $S \cup\{p\} \rightarrow \tau+1$.

The symbol " $\leq$ " often will be used simultaneously as the order relation on a given ordered space as well as the order relation on the ordinals. However, no ambiguity should arise.

Received by the editors February 9, 1987 and, in revised form, October 21, 1988.

1980 Mathematics Subject Classification (1985 Revision). Primary 54F05, 06A05, 54F99; Secondary 54A20, 03E10, 54D15, 54E20, 54B15.

Key words and phrases. Totally orderable, suborderable, scattered, strongly collectionwise Hausdorff, ordering property, ordinal property, stationary set, interlacing property, sequence, length, $Q$-gap, greatest ordered compactification, monotone normality, far point.

The author is pleased to thank the Academies of Sciences of Czechoslovakia, Hungary, the United States and the International Research and Exchanges Board for support during the preparation of this paper. 
If an element $p$ of an orderable space $X$ is the limit of two disjoint closed sequences in $X-\{p\}$, when is there an admissible order on $X$ such that the sequences are interlaced, i.e are on the same side of $p$ ? If $X$ is compact, it is necessary and sufficient for both sequences to have countable cardinality. In general a new property is needed. (Also see Lemma 4.)

A point $p$ in a space $X$ satisfies the interlacing property if $p$ is not the limit of two disjoint closed sequences in $X-\{p\}$ where either (1) the sequences are of different cardinalities, or (2) one sequence is homeomorphic to a stationary subset of an uncountable regular ordinal.

Note in an orderable space if a point is a limit of disjoint sequences $S$ and $T$ of the same uncountable cardinality $\tau$, and $S$ and $T$ are interlaced with respect to some admissible order where $T$ is homeomorphic to a stationary subset of $\tau$, then $\bar{S} \cap T \neq \varnothing$. Also note if a sequence is homeomorphic to a stationary set, then no final segment is paracompact. This follows easily from [EL].

In the induction proof of Theorem 1 in $\left[\mathrm{P}_{1}\right]$ an admissible order is found for some open set $O$ as an extension of an order implied by the induction on each member of a partition of $O$. This induction proceeds using the properties given in the theorem for a neighborhood subbase of a point. Using this proof in the noncompact case, it is possible that some nonconvergent sequence converges in the order topology induced by the order found on $O$. So another property is needed generalizing the conditions in Theorem 1 in $\left[\mathrm{P}_{1}\right]$ on a neighborhood subbase at a point.

Let $\mathscr{I}=\left\{I_{\alpha}\right\}_{\alpha<\tau}$ be a decreasing nest of clopen sets in a space, $\tau$ a regular ordinal. $\mathscr{I}$ has the ordering property if for each nonzero limit ordinal $\beta<\tau$, $\bigcap_{\alpha<\beta} I_{\alpha}$ has at most one boundary point $i_{\beta}$ where

(1) $\left\{I_{\xi}-\right.$ int $\left.\bigcap_{\alpha<\beta} I_{\alpha}: \xi<\beta\right\}$ is a neighborhood base of $i_{\beta}$ in $I_{0}-\operatorname{int} \bigcap_{\alpha<\beta} I_{\alpha}$; and

(2) $i_{\beta}$ satisfies the interlacing property in $\bigcap_{\alpha<\beta} I_{\alpha}$.

There is another problem in the induction procedure.

The length of a scattered space $X, l(X)$, is the least ordinal $\alpha$ such that the $\alpha$ th derived set, $X^{(\alpha)}$, of $X$ is empty. In $\left[\mathrm{P}_{1}\right] l(X)$ is a nonlimit ordinal $\lambda+1$, and $X$ is partitioned into a pairwise disjoint collection $\left\{O_{p}: p \in X^{(\lambda)}\right\}$ of clopen sets such that $O_{p} \cap X^{(\lambda)}=\{p\}$ for each $p \in X^{(\lambda)}$. Then the induction hypothesis is used to order each $O_{p}$, and without loss of generality it is assumed that $\left|X^{(\lambda)}\right|=1$.

For a noncompact scattered space of length a nonlimit ordinal, it may be impossible to get such a partition. A space is strongly collectionwise Hausdorff if for each closed discrete subspace $Y$, there is a discrete family of open sets $\left\{U_{y}: y \in Y\right\}$ such that $y \in U_{y}$. Every orderable space is strongly collectwise Hausdorff. Let $X$ be the space $\omega_{1} \times\left(\omega_{0}+1+\omega_{1}^{*}\right)-\{\langle\alpha, \beta\rangle: \alpha$ a limit ordinal, $\left.\beta \geq \omega_{0}\right\}$, where the product is Cartesian, and $\omega_{1}^{*}$ is $\omega_{1}$ with its order reversed. 
Now $l(X)=\omega_{1}+1$, but the points of $X^{\left(\omega_{1}\right)}=\left\{\left\langle\alpha, \omega_{0}\right\rangle: \alpha\right.$ a nonlimit ordinal $\}$ cannot be separated by a discrete family of open sets. $X$ is even hereditarily collectionwise Hausdorff and satisfies conditions 2 and 3 of Theorem 1.

If the length $\alpha$ of a scattered space is a limit ordinal, the induction hypothesis could be used if the space could be partitioned into pairwise disjoint clopen sets each of length less than $\alpha$. This is precisely the case for paracompact scattered spaces (see $[\mathrm{T}]$ ). In general as in $\left[\mathrm{P}_{2}\right]$, the space can be partitioned into a pairwise disjoint collection of clopen sets each satisfying a paracompactness-like property away from what corresponds to a special kind of end gap of an ordered space. To use the induction hypothesis for ordering a member of this partition, a property is needed for a "neighborhood base" of such a gap analogous to the condition satisfied for a neighborhood subbase of a point.

Then in condition $3 b$ of Theorem 1 in the next section, the nest is a neighborhood base of a gap. The length restriction and ordering property on the nest permits the induction to be performed. These gaps of a space $X$ are actually points of $\beta X-X$ which are not in the closure in $\beta X$ of any closed discrete subset of $X$, i.e., far points of $X$. The union of $X$ and the set of these "gap points" in $\beta X-X$ is a paracompact scattered space (which is $v X$ if $X$ has no closed discrete subset of measurable cardinality) satisfying condition 2 , which is enough to be orderable.

\section{RESULTS}

Theorem 1. A scattered space $X$ is orderable iff

(1) $X$ is strongly collectionwise Hausdorff;

(2) for each nonisolated $p \in X$ there is a neighborhood subbase $\left\{L_{\alpha}\right\}_{\alpha<\tau} \cup$ $\left\{R_{\alpha}\right\}_{\alpha<\gamma}$, the union of two nests each with the ordering property; and

(3) for each clopen $Y \subseteq X$ where $l(Y)$ is a limit ordinal, $Y=\bigoplus_{r \in R} O_{r}$ such that for each $r$

(a) $l\left(O_{r}\right)<l(Y)$, or

(b) in $O_{r}$ there is a nest $\left\{I_{\alpha}\right\}_{\alpha<\tau}$ with the ordering property where $\bigcap_{\alpha<\tau} I_{\alpha}=$ $\varnothing$ and $l\left(O_{r}-I_{\alpha}\right)<l(Y)$ for all $\alpha<\tau$.

The proof is given after some preliminaries.

A left gap in an ordered space $X$ is a nonempty clopen initial convex set in $X$ and has no maximum. A left gap is a left $Q$-gap [GHen] if it contains a closed discrete subset cofinal in the gap. A right gap and right $Q$-gap are defined analogously. The greatest ordered compactification $b X$ of $X[\mathrm{~F}]$ is the compactification whose growth is order-isomorphic to the set of gaps of $X$. Hence, to each left (right) gap $A$ in $X$ corresponds the gap point $p_{A}$ in $b X-X$, which is the sup (inf) in $b X$ of $A$.

The following lemma considers the set $S$ of "highest level" non- $Q$-gap points of a scattered ordered space.

Lemma $1\left[\mathrm{P}_{2}\right]$. Let $X$ be a scattered ordered space of length $\lambda$, where $\lambda$ is a limit ordinal. Let $S$ be the set of all non-Q-gap points $p_{A}$ of $X$ such that $p_{A}$ 
is a boundary point of $A^{(\xi)}$ for each $\xi<\lambda$. Then $S$ is discrete and closed in $X \cup S$.

In the proof of the theorem in $\left[\mathrm{P}_{2}\right]$ it was implied that the next lemma may not be true.

Lemma 2. Let $X$ be an ordered space and $X^{*}=X \cup\{p: p \in b X-X$ and $p$ is a non-Q-gap point of $X\}$. Then $X^{*}$ is paracompact.

Proof. In [GHen] it is shown that an ordered space is paracompact iff all its gaps are $Q$-gaps. The same proof holds for a subspace of an ordered space. So it is sufficient to show each point of $b X-X$ which is a $Q$-gap point of $X$ is also a $Q$-gap point of $X^{*}$. So let $p$ be a $Q$-gap point of $X$. Then there is a monotone sequence $\left\{x_{\alpha}\right\}_{\alpha<\tau}$ in $X$ converging to $p$, where $\left\{x_{\alpha}: \alpha<\tau\right\}$ is closed and discrete in $X$ and $\tau$ is a limit ordinal. By the definition of a $Q$-gap, $\left\{x_{\alpha}: \alpha<\beta\right\}$ converges to a $Q$-gap point of $X$ for each limit ordinal $\beta<\tau$. Hence $\left\{x_{\alpha}: \alpha<\tau\right\}$ is closed and discrete in $X^{*}$. So $p$ is a $Q$-gap point of $X^{*}$.

The proof of the next lemma is simple.

Lemma 3. A sequence is homeomorphic to a stationary set iff it contains no cofinal, discrete, closed subset.

The following generalizes the lemma in $\left[\mathrm{P}_{1}\right]$.

Lemma 4. Let $X$ be a scattered orderable space, and let $p \in X$ satisfy the interlacing property. Then there is an admissible order on $X$ with $p$ as its first point.

Proof. By $\left[\mathrm{P}_{2}\right]$ there is an admissible order $\leq$ with respect to which $X$ has a first point. If $p$ is the first point of $X$, we are done. Otherwise if $p$ is not an element of the closure of the initial open segment $(-\infty, p)$, then $p$ has an immediate predecessor $p^{\prime}$. Let $X_{1}=\left(-\infty, p^{\prime}\right]$ and $X_{2}=[p, \infty)$.

Case 1. $X$ has a last point. Then define a new order $\leq$ on $X$ so that $\leq$ and $\leq^{\prime}$ have identical restrictions to $X_{1}$ and $X_{2}$, and $X_{2} \leq X^{\prime} X_{1}$. (Define $A \leq B$ if $a \leq^{\prime} b$ for every $a \in A$ and $b \in B$.)

Case 2. $X$ has no last point. Since $X_{2}$ is scattered, it has an isolated point $q$ with an immediate predecessor $q^{\prime}$. Let $X_{21}=\left[p, q^{\prime}\right]$ and $X_{22}=[q, \infty)$. Define $\leq$ so that $\leq$ and $\leq$ ' have identical restrictions to $X_{1}$ and $X_{2}$, and $X_{21} \leq^{\prime} X_{1} \leq^{\prime} X_{22}$.

Then in each of the above cases $\leq$ satisfies the lemma.

With respect to $\leq$ if $p$ is the last point of $X$, or $p \notin \operatorname{cl}(p, \infty)$, then by first reversing the order $\leq$, an analogous argument produces an order satisfying the lemma.

Finally, suppose that $\{p\}=\operatorname{cl}(-\infty, p) \cap \operatorname{cl}(p, \infty)$. Then converging to $p$ are sequences $S$ and $T$ of the same cardinality closed in $X-\{p\}$, where $S \leq p \leq$ 
$T$, and neither sequence is homeomorphic to a stationary set. Then by Lemma $3, S$ and $T$ contain closed (in $X-\{p\}$ ), discrete, monotone subsequences $\left\{s_{\alpha}\right\}_{\alpha<\tau}$ and $\left\{t_{\alpha}\right\}_{\alpha<\tau}$ respectively.

Case 1. $\tau \neq \omega_{0}$. For each $\alpha<\tau$ let $\alpha^{\prime}$ be the $\alpha$ th limit ordinal, $S_{\alpha}=\{x \in$ $X: s_{\beta}<x<s_{\gamma}$ for all $\beta<\alpha^{\prime}$ and for some $\gamma$ where $\left.\alpha^{\prime}<\gamma<(\alpha+1)^{\prime}\right\}$, and $T_{\alpha}=\left\{x \in X: t_{\gamma}<x<t_{\beta}\right.$ for all $\beta<\alpha^{\prime}$ and for some $\gamma$ where $\alpha^{\prime}<\gamma<$ $\left.(\alpha+1)^{\prime}\right\}$. Then for all $\alpha, S_{\alpha}$ and $T_{\alpha}$ are clopen convex sets, $S_{0}$ has a first but no last point, $T_{0}$ has no first point, $S_{\alpha}$ and $T_{\alpha}$ have neither a first nor last point for $\alpha \neq 0$, and $\left\{S_{\alpha}: \alpha<\tau\right\} \cup\left\{T_{\alpha}: \alpha<\tau\right\}$ partitions $X-\{p\}$. Then define a new order $\leq^{\prime}$ on $X$ so that for all $\alpha<\tau,\{p\} \leq^{\prime} S_{\beta} \leq^{\prime} T_{\alpha} \leq^{\prime} S_{\alpha}$ for all $\beta>\alpha$; for $\alpha \neq 0, \leq$ and $\leq^{\prime}$ have identical restrictions to $T_{0}, S_{\alpha}$, and $T_{\alpha}$; and the restriction of $\leq$ to $S_{0}$ is identical to the restriction of $\leq$ to $S_{0}$ if $T_{0}$ has a last point, and is the reverse order of the restriction of $\leq$ to $S_{0}$ if $T_{0}$ has no last point.

Case 2. $\tau=\omega_{0}$. Then we may assume the points of $S$ and $T$ are isolated. For each $n<\omega_{0}$ let $S_{0}=\left\{x \in X: x<s_{0}\right\}, S_{n+1}=\left[s_{n}, s_{n+1}\right), T_{0}=\left\{x \in X: t_{0}<\right.$ $x\}$, and $T_{n+1}=\left(t_{n+1}, t_{n}\right]$. Then $S_{n}$ and $T_{n}$ are clopen; $S_{0}, S_{n+1}$, and $T_{n+1}$ have first and last points; $T_{0}$ has a first point; and $\left\{S_{n}: n<\omega_{0}\right\} \cup\left\{T_{n}: n<\omega_{0}\right\}$ partitions $X-\{p\}$. Define $\leq^{\prime}$ on $X$ so that $\{p\} \leq^{\prime} T_{n+1} \leq^{\prime} S_{n} \leq^{\prime} T_{n}$, and $\leq^{\prime}$ and $\leq$ have identical restrictions to $S_{n}$ and $T_{n}$.

Then in each of these cases $\leq$ ' satisfies the lemma.

Proof of Theorem 1. For necessity every orderable space is strongly collectionwise Hausdorff. As in the proof of Theorem 1 in $\left[\mathrm{P}_{1}\right]$, for a given admissible order on $X$ and each nonisolated $p \in X$, there are decreasing clopen nests $\left\{L_{\alpha}\right\}_{\alpha<\tau}$ of left rays and $\left\{R_{\alpha}\right\}_{\alpha<\gamma}$ of right rays whose union forms a neighborhood subbase for $p$, and hence satisfies condition (2) of the theorem.

Now let $Y$ be a clopen subset of $X$ of length a limit ordinal $\lambda$. By $\left[\mathrm{P}_{2}\right]$ $Y$ is orderable. So fix an admissible order on $Y$. Let $S$ be the set of highest level non- $Q$-gap points of $Y$ described in Lemma 1 . The argument is similar to and cleans up the proof of the theorem in $\left[\mathrm{P}_{2}\right]$. If $S=\varnothing$, the proof that $Y$ satisfies condition (3) follows the argument below for $\left(-\infty, y_{0}\right]$. So assume $S \neq \varnothing$. Since $S \cup Y$ is strongly collectionwise Hausdorff and 0 -dimensional, it is the disjoint union of clopen subsets each containing one point of $S$. So we may assume $S=\{u\}$, and $u$ is an endpoint, say largest point, of $S \cup Y$. (Note no point of $S$ is in both the closures of the points less than it and the points greater than it.)

There is an increasing sequence $\left\{y_{\alpha}\right\}_{\alpha<\tau}$ of isolated points in $Y$ converging to $u$, where $\tau$ is a regular ordinal. For each $\alpha<\tau$, let $I_{\alpha}$ be the open interval $\left(y_{\alpha}, u\right)$. Then $\left\{I_{\alpha}\right\}_{\alpha<\tau}$ satisfies the ordering property in $Y$, and $\bigcap_{\alpha<\tau} I_{\alpha}=\varnothing$. For some $\alpha_{0}<\tau, l\left(\left(y_{\alpha_{0}}, y_{\alpha}\right]\right)<\lambda$ for all $\alpha>\alpha_{o}$. Otherwise for some cofinal subset $C$ of $\tau, l\left(\left(y_{\alpha}, y_{\alpha}^{\prime}\right]\right)=\lambda$, where $\alpha^{\prime}$ is the immediate successor of $\alpha$ in 
$C$ for each $\alpha \in C$; then since $u$ is a non- $Q$-gap of $Y,\left\{y_{\alpha}: \alpha \in C\right\}$ must have a boundary point $p \in Y$; so $p \in Y^{(\lambda)}=\varnothing$, which is impossible. So without loss os generality let $\alpha_{0}=0$. Note $I_{0}-I_{\alpha}=\left(y_{0}, y_{\alpha}\right]$ for each $\alpha>0$. Then $\left\{I_{\alpha}\right\}_{\alpha<\tau}$ in $I_{0}$ satisfies condition $(3)(\mathrm{b})$.

Now let $I$ be the ray $\left(-\infty, y_{0}\right]$ and $I^{*}$ as in Lemma 2. So $I^{*}$ is paracompact. For each $\alpha<\lambda$ let $\mathscr{C}_{\alpha}$ be the collection of all maximal convex in $X$ subsets of $I-I^{(\alpha)}$, and for each $C \in \mathscr{C}_{\alpha}$ let $C^{\prime}=\mathrm{cl}_{I^{*}} C-\left(\left(\mathrm{cl}_{X} C\right)-C\right)$. (Note $\left(\mathrm{cl}_{X} C\right)-C$ consists of at most two points.) Then $C^{\prime}$ is open and convex in $I^{*}$. Let $\mathscr{C}_{\alpha}^{\prime}=\left\{C^{\prime}: C \in \mathscr{C}_{\alpha}\right\}$. Then $\bigcup_{\alpha<\lambda} \mathscr{C}_{\alpha}^{\prime}$ is an open cover of $I^{*}$. So it has a locally finite open refinement $\mathscr{F}$ which - since Ind $I^{*}=0$ - can be chosen to be a clopen partition of $I^{*}$. Note for each $F \in \mathscr{F}, l(F \cap I)<\lambda$. Hence since $I$ is clopen in $Y, Y=I_{0} \oplus \bigoplus_{F \in \mathscr{F}}(F \cap I)$. So condition (3) is satisfied.

The sufficiency proof, similar to the one for Theorem 1 in $\left[\mathrm{P}_{1}\right]$, is by induction on $\lambda=l(x)$. So assume the theorem holds for all scattered spaces of length $<\lambda$.

If $\lambda$ is a nonlimit ordinal, $X$ can be partitioned into clopen sets each containing one point of $X^{(\lambda-1)}$ since $X$ is strongly collectionwise Hausdorff and 0 -dimensional. Then since by $\left[\mathrm{P}_{2}\right]$ every suborderable scattered space is orderable, without loss of generality let $X^{(\lambda-1)}$ be a singleton $\{q\}$. There is a neighborhood subbase $\left\{L_{\alpha}\right\}_{\alpha<\tau} \cup\left\{R_{\alpha}\right\}_{\alpha<\gamma}$ of $q$ satisfying condition (2) in the theorem.

For each $\beta<\tau$ let $L_{\beta}^{\prime}=\bigcap_{\alpha<\beta} L_{\alpha}-L_{\beta}$. Since $L_{\beta}^{\prime}$ is closed, it is strongly collectionwise Hausdorff. Since $L_{\beta+1}^{\prime}$ is clopen, it satisfies condition (3) of the theorem. Since for $\beta$ a limit ordinal $L_{\beta}^{\prime}$ has at most one boundary point and $X$ is 0 -dimensional, each relatively clopen subset $C$ of $L_{\beta}^{\prime}$ has a clopen neighborhood $V$ in $X$, where $C=L_{\beta}^{\prime} \cap V$ and $l(V)=l(C)$. So $L_{\beta}^{\prime}$ also satisfies condition (3). Hence the induction hypothesis can be applied to $L_{\beta}^{\prime}$ since $l\left(L_{\beta}^{\prime}\right)<\lambda$. The same is true for the analogously defined $R_{\beta}^{\prime}$ for all $\beta<\gamma$.

Now the sufficiency proof of Theorem 1 in $\left[\mathrm{P}_{1}\right]$ can be used by applying Lemma 4 , and the fact that $\left\{L_{\alpha}\right\}_{\alpha<\tau}$ and $\left\{R_{\alpha}\right\}_{\alpha<\gamma}$ satisfy the ordering property.

Note the order constructed on $X$ may only be an admissible suborder. (That is, there is an order preserving imbedding of $X$ with this order into an ordered space. The points which may not have open interval neighborhood bases are the nonboundary endpoints of the $L_{\alpha}^{\prime}$ and $R_{\alpha}^{\prime}$.) However by $\left[\mathrm{P}_{2}\right]$ this is all right.

Finally if $\lambda$ is a limit ordinal, then $X=\bigoplus_{r \in R} O_{r}$ where for each $r, O_{r}$ satisfies condition (3)(a) or (b). Fix $r \in R$. If $O_{r}$ satisfies condition (3)(a), then by the induction hypothesis it is orderable. If $O_{r}$ satisfies condition $(3)(\mathrm{b})$, then for each $\beta<\tau$ let $I_{\beta}^{\prime}=\bigcap_{\alpha<\beta} I_{\alpha}-I_{\beta}$. Then using the argument of the case where $\lambda$ is nonlimit, an admissible suborder is constructed on $O_{r}$. Since this can be done for every element of $R$, then by $\left[\mathrm{P}_{2}\right] X$ is orderable. 
Consider the following strengthening of the ordering property. Let $\mathscr{I}=$ $\{I\}_{\alpha<\tau}$ be a decreasing nest of clopen sets in a space, $\tau$ a regular ordinal. $\mathscr{I}$ has the ordinal property if for each nonzero limit ordinal $\beta<\tau, \bigcap_{\alpha<\beta} I_{\alpha}-I_{\beta}$ contains at most one point $i_{\beta}$, where $\left\{I_{\alpha}-I_{\beta}: \alpha<\beta\right\}$ is a neighborhood base of $i_{\beta}$.

The ordinal property is a strengthening of Baker's property $\mathrm{D}[\mathrm{B}]$ and the ordering property.

The next result generalizes Baker's characterization [B] of compact ordinal spaces.

Theorem 2. A scattered space $X$ is homeomorphic to a subspace of an ordinal space iff

(1) $X$ is strongly collectionwise Hausdorff;

(2) for each nonisolated $p \in X$ there is a neighborhood base with the ordinal property; and

(3) condition (3) of Theorem 1 with ordering property replaced by ordinal property.

Proof. The necessity proof of Theorem 1 works here noticing that the nests constructed in that proof satisfy the ordinal property, and here in the admissible order on $X$ no point should be the limit of a decreasing sequence. (Actually the only reason for reordering $X$ is that many of the concepts were defined here only for ordered spaces.)

For sufficiency let $X$ satisfy conditions (1), (2), and (3). Assume Theorem 2 holds for all scattered spaces of length $<l(X)=\lambda$. Let $\mathscr{I}=\left\{I_{\alpha}\right\}_{\alpha<\tau}$ be a collection of subsets of $X$ with the ordinal property such that for all $\alpha<\tau$ $l\left(I_{0}-I_{\alpha}\right)<l(X)$. As in the proof of Theorem $1, I_{\beta}^{\prime}=\bigcap_{\alpha<\beta} I_{\alpha}-I_{\beta}$ satisfies the induction hypothesis for each $\beta<\tau$; so there is a well ordering $\leq_{\beta}$ of $I_{\beta}^{\prime}$ under which $I_{\beta}^{\prime}$ is a well-ordered GO space (i.e., there is an order preserving map into an ordinal space), and for $\beta$ a nonzero limit ordinal the boundary point, if any, of $I_{\beta}^{\prime}$ is the first point since it is isolated in $I_{\beta}^{\prime}$. Define a well order $\leq$ on $I_{0}-\bigcap_{\alpha<\tau} I_{\alpha}$ as follows: for each $\alpha<\beta<\tau, I_{\alpha}^{\prime}<I_{\beta}^{\prime}$, and $\leq$ restricted to $I_{\alpha}^{\prime}$ is $\leq_{\alpha}$. Since $\mathscr{I}$ has the ordinal property, $I_{0}-\bigcap_{\alpha<\tau} I_{\alpha}$ is a well-ordered GO space under $\leq$. That is, $I_{0}-\bigcap_{\alpha<\tau} I_{\alpha}$ is homeomorphic to a subspace of an ordinal space. Moreover, if $\mathscr{I}$ is a neighborhood base of a nonisolated point $p \in X$, then $\leq$ can be extended to $I_{0}$ such that $p$ is the last point since $\bigcap_{\alpha<\tau} I_{\alpha}=\{p\}$.

Using this fact and noting the disjoint union of well-ordered GO spaces is homeomorphic to a subspace of an ordinal space, the sufficiency proof here exactly follows the pattern of the sufficiency proof of Theorem 1 .

Example 1. There is an orderable scattered space which is not homeomorphic to a subspace of an ordinal space even though each of its points has a neighborhood base with the ordinal property. 
Let $X$ be the lexicographic product $\omega_{1} \times\left(\omega_{0}+1\right)^{*}$, where $\left(\omega_{0}+1\right)^{*}$ is $\omega_{0}+1$ with its order reversed. Since $X$ is first countable, each of its points has a neighborhood base with the ordinal property. As in the proof in $\left[\mathrm{P}_{1}\right]$ that the example in $\S 2.1$ is not orderable, there is no well order of $X$ under which $X$ is a $\mathrm{GO}$ space. This follows from the pressing down lemma since $\left\{\left\langle\alpha, \omega_{0}^{*}\right\rangle: \alpha<\omega_{1}\right\} \quad\left(\omega_{0}^{*}\right.$ the first point of $\left.\left(\omega_{0}+1\right)^{*}\right)$ is homeomorphic to the stationary set $\omega_{1}$.

G. Moran [M] proved a Hausdorff space is compact, scattered, and orderable iff it is the 2 to 1 continuous image of a compact ordinal space. Such a map is a quotient map. In fact it is closed. There is a 2 to 1 Hausdorff quotient (but not a closed map) of $\omega_{0}^{2}$ which has no isolated points: just identify points via a 1 to 1 correspondence between isolated and nonisolated points. However, we have the following

Theorem 3. A space is scattered and orderable iff it is the 2 to 1 image under a closed map of a subspace of an ordinal space.

The proof is given after some lemmas.

Lemma $5\left[\mathrm{KR}_{1}\right]$. The class of scattered spaces is invariant under perfect maps.

Lemma 6. Let $f: p \rightarrow X$ be a 2 to 1 , closed, onto map, where $P$ is a subspace of an ordinal space. Then there is a 2 to 1 , closed, onto map $g: P^{*} \rightarrow X^{\prime}$ such that $g \mid P=f, X^{\prime} \subset \beta X$, and $P^{*}=P \cup\{p: p \in b P-P$ and $p$ is a non-Q-gap point of $P\}$.

Proof. In [GHen] it is shown that $P^{*} \subset \beta P$. Let $f_{\beta}: \beta P \rightarrow \beta X$ be the unique extension of $i \cdot f$, where $i: X \rightarrow \beta X$ is the inclusion map. Let $g=f_{\beta} \mid P^{*}$, and $X^{\prime}=g\left(P^{*}\right)$.

It is first shown that $f_{\beta}$ is 2 to 1 . Since $P$ is normal and $f$ is perfect, $X$ is normal. Hence, disjoint $X$-closed sets have disjoing closures in $\beta X$.

Suppose $f_{\beta}\left(p_{1}\right)=f_{\beta}\left(p_{2}\right)=f_{\beta}\left(p_{3}\right)$, where $p_{1}, p_{2}$, and $p_{3}$ are distinct points in $\beta P$. Then there are mutually disjoint closed neighborhoods $P_{i}$ of $p_{i}, I=$ $1,2,3$. Hence $f\left(P_{i} \cap P\right) \cap f\left(P_{j} \cap P\right) \neq \varnothing$ for any $i \neq j$ since $f$ is a closed map. Let $A=P \cap f^{-1}\left(f\left(P_{1} \cap P\right) \cap f\left(P_{2} \cap P\right)\right)$. Then $A$ is closed in $P$, and $p_{1} \in \mathrm{cl}_{\beta P} A$. So $f(A) \cap f\left(P_{3} \cap P\right) \neq \varnothing$. Therefore, for $x \in f(A) \cap f\left(P_{3} \cap P\right)$ we have $\left|f^{-1}(x)\right|>2$, which contradicts $f$ being 2 to 1 . Hence, $f_{\beta}$ is 2 to 1 .

It will be shown that $g$ is closed by showing $f_{\beta}^{-1} f_{\beta}\left(P^{*}\right)=P^{*}$. Since $f$ is perfect, $f_{\beta}(\beta P-P) \subset \beta X-X$ (see [E, Theorem 3.7.15, p. 239]). Let $p \in P^{*}-P$. So we need only show $f_{\beta}^{-1} f_{\beta}(p) \subset P^{*}$.

Let $\left\{p_{\alpha}\right\}_{\alpha<\tau}$ be a $P$-closed strictly increasing sequence (of course, there are no infinite decreasing sets in a well-ordered set) converging to $p$, where $\tau$ is a regular ordinal. Consider the only nontrivial case where $f_{\beta}^{-1} f_{\beta}(p)=\{p, q\}$ and $p \neq q$. Let $N$ be a closed neighborhood in $\beta P$ of $q$ which misses $p$. Let $A$ be the first $\tau$ elements of (the subspace of an ordinal space) $N \cap$ 
$f^{-1} f\left(\left\{p_{\alpha}: \alpha<\tau\right\}\right)$. Since $f$ is a closed map, $A$ is closed in $P$ and $q^{\prime}=$ $\sup _{b P} A$ is a gap point of $P$. Since $p$ is a non- $Q$-gap point of $P,\left\{p_{\alpha}: \alpha<\tau\right\}$ contains no $P$ closed, discrete, cofinal subset. Hence since $f$ is closed and 2 to $1, A$ contains no $P$ closed, discrete, cofinal subset. So there is no $P$ closed, discrete set cofinal in the $P$ gap $\left\{p^{\prime} \in P: p^{\prime}<q^{\prime}\right\}$. Hence, $q^{\prime} \in P^{*}-P$ and $f_{\beta}\left(q^{\prime}\right)=f_{\beta}(p)$. Since $f_{\beta}$ is 2 to 1 and $q^{\prime} \neq p$, then $q^{\prime}=q \in P^{*}$. Therefore, $f_{\beta}^{-1} f_{\beta}(p) \subset P^{*}$.

Lemma 7. Let $X$ be the image under a 2 to 1 closed map of a paracompact subspace of an ordinal space. Then $X$ is scattered and orderable.

Proof. $X$ is scattered by Lemma 5. Assume this lemma is true for every scattered space of length $<l(X)=\lambda$.

It will be shown that $X$ satisfies Theorem 1. As the image under a closed map of a paracompact space, $X$ is paracompact. So $X$ satisfies conditions (1) and (by [T]) (3)(a) of Theorem 1 . So we need only show $X$ satisfies condition (2).

If $\lambda$ is a limit ordinal, then by [T] $X=\bigoplus_{r \in R} O_{r}$, where for each $r \in$ $R, l\left(O_{r}\right)<l(X)$; and so by the induction hypothesis $O_{r}$ is orderable. So $X$ is suborderable, and by $\left[\mathrm{P}_{2}\right] X$ is orderable.

Now assume $\lambda$ is a nonlimit ordinal. By [T] $X=\bigoplus_{r \in R} O_{r}$, where for each $r \in R, O_{r}$ contains exactly one point of $X^{(\lambda-1)}$. By [ $\left.\mathrm{P}_{2}\right]$ if each $O_{r}$ is orderable, then $X$ is orderable. So without loss of generality let $X^{(\lambda-1)}=\{x\}$.

By the induction hypothesis we need only show condition (2) of Theorem 1 is satisfied at $x$. Let $f: P \rightarrow X$ be a 2 to 1 , closed, onto map where $P$ is a paracompact subset of an ordinal space. The proof is actually the proof of Corollary 2 in $\left[\mathrm{P}_{1}\right]$ with the following additions.

$X$ is 0 -dimensional by [T] since it is paracompact and scattered. Since $f$ is closed, $f(0)$ is a neighborhood of $x$ for any neighborhood $O$ of $f^{-1}(x)$ (see [E, Theorem 1.4.12, p. 52]).

First suppose $f^{-1}(x)$ is a singleton $\{\delta\}$. Then since $f$ is closed and $X$ is 0 -dimensional, there is a closed increasing sequence $\left\{\xi_{\alpha}\right\}_{\alpha<\tau} \rightarrow \delta$ (assuming $\delta \in P^{(1)}$; otherwise $x$ is isolated), and for all $\alpha<\alpha^{\prime}<\tau$ a clopen neighborhood $L_{\alpha}$ of $x$ such that $\left(\xi_{\alpha}, \delta\right] \supset f^{-1}\left(L_{\alpha}\right) \supset\left(\xi_{\alpha^{\prime}}, \delta\right.$ ] (these intervals being in $P$, not the ordinal superspace).

Hence since $\left\{f^{-1}\left(L_{\alpha}\right)\right\}_{\alpha<\tau}$ is a nested neighborhood base of $\delta,\left\{L_{\alpha}\right\}_{\alpha<\tau}$ is a nested neighborhood base of $x$.

For a limit ordinal $\beta<\tau$ if $\left\{\xi_{\alpha}\right\}_{\alpha<\beta}$ does not converge in $X$, then using the argument in the proof of Corollary 2 in $\left[P_{1}\right]$ that $\bigcap_{\alpha<\beta} L_{\alpha}$ has at most one boundary point, $\bigcap_{\alpha<\beta} L_{\alpha}$ has no boundary point since $f$ is closed. (In this and the next paragraph it might be helpful to note that since $f$ is closed, $y \in$ int $\bigcap_{\alpha<\beta} L_{\alpha}$ iff $f^{-1}(y) \subset$ int $f^{-1}\left(\bigcap_{\alpha<\beta} L_{\alpha}\right)=\operatorname{int} \bigcap_{\alpha<\beta}\left(\xi_{\alpha}, \delta\right]$.)

So now let $\left\{\xi_{\alpha}\right\}_{\alpha<\beta} \rightarrow \xi_{\beta}$. Then $f\left(\xi_{\beta}\right)$ is the unique boundary point of $\bigcap_{\alpha<\beta} L_{\alpha}$. To show that $\left\{L_{\alpha}\right\}_{\alpha<\tau}$ satisfies the ordering property at $f\left(\xi_{\beta}\right)$ first 
note int $\bigcap_{\alpha<\beta} L_{\alpha}=\bigcap_{\alpha<\beta} L_{\alpha}-f\left(\xi_{\beta}\right)=f\left(\left[\xi_{\beta}, \delta\right]\right)-f\left(\xi_{\beta}\right)$, and by construction $f^{-1} f\left(\left[\xi_{\beta}, \delta\right]\right)=\left[\xi_{\beta}, \delta\right]$; hence $\left\{L_{\xi}-\operatorname{int} \bigcap_{\alpha<\beta} L_{\alpha}: \xi<\beta\right\}$ is a neighborhood base of $f\left(\xi_{\beta}\right)$ in $L_{0}-\operatorname{int} \bigcap_{\alpha<\beta} L_{\alpha}$ since $\left\{f^{-1}\left(L_{\xi}-\operatorname{int} \bigcap_{\alpha<\beta} L_{\alpha}\right): \xi<\beta\right\}$ is a neighborhood base of $f^{-1} f\left(\xi_{\beta}\right)$ in $f^{-1}\left(L_{0}-\right.$ int $\left.\bigcap_{\alpha<\beta} L_{\alpha}\right)$. As in the proof in Corollary 2 in $\left[\mathrm{P}_{1}\right], f\left(\xi_{\beta}\right)$ satisfies the interlacing property in $\bigcap_{\alpha<\beta} L_{\alpha}$ noting that if $f^{-1}\left(f\left(\xi_{\beta}\right)\right)=\left\{\xi_{\beta}, \xi\right\}$ and $f\left(\xi_{\beta}\right)$ is the limit in $\bigcap_{\alpha<\beta} L_{\alpha}$ of a sequence $T$ homeomorphic to a stationary set, then every closed cofinal subset of $(-\infty, \xi)$ intersects $f^{-1}(T)$. Therefore, condition (2) of Theorem 1 is satisfied for $f^{-1}(x)$ a singleton.

If $f^{-1}(x)$ consists of two points, then analogously condition (2) of Theorem 1 is satisfied using the above additions to the proof of Corollary 2 in $\left[\mathrm{P}_{1}\right]$ replacing in that proof 0 and $\lambda$ by $-\infty$ and $\infty$ respectively.

Proof of Theorem 3. For necessity let $X$ be an orderable scattered space. By $\left[\mathrm{P}_{2}\right] X$ has an orderable scattered compactification $s X$. By [M] there is a 2 to 1 onto map $f: \lambda \rightarrow s X$, where $\lambda$ is a compact ordinal space. Then $f \mid f^{-1}(X): f^{-1}(X) \rightarrow X$ is a closed, onto, 2 to 1 map.

Since by $\left[\mathrm{P}_{2}\right]$ every suborderable scattered space is orderable, sufficiency follows from Lemmas 6 and 7.

Under what kind of maps is the length condition of a scattered range determined by its scattered domain?

For a point $p$ in a scattered space $P$, the length of $p, l(p)$, equals an ordinal $\alpha$ if $p \in P^{(\alpha)}-P^{(\alpha+1)}$.

Proposition. Let $f: P \rightarrow X$ be a closed onto map with discrete fibers such that $P$ and $X$ are scattered. For each $x \in X, l(x)=\sup \left\{l(p): p \in f^{-1}(x)\right\}$.

Proof. Let $x \in X$. Assume the lemma is true for all $y \in X$ where $l(y)<$ $l(x)=\lambda$.

Let $O$ be a neighborhood of $f^{-1}(x)$. Since $f$ is a closed map, there exists a neighborhood $N$ of $x$ such that $f^{-1}(N) \subset O$. Moreover, $N$ can be chosen such that $N \cap X^{(\lambda)}=\{x\}$. For each $\alpha<\lambda$, there exists $y \in N$ such that $l(y)=\alpha$. Hence, by the induction hypothesis $\sup \left\{l(p): p \in f^{-1}(y)\right\}=\alpha$. Hence, $\sup \left\{l(p): p \in f^{-1}(x)\right\} \geq \lambda$.

On the other hand, since $f^{-1}(x)$ is discrete, we have for any $p \in f^{-1}(x)$ a neighborhood $O_{p}$ such that $O_{p} \cap f^{-1}(x)=\{p\}$ and $O_{p} \subset f^{-1}(N)$. So for any $q \in O_{p}-\{p\}, f(q) \in N-\{x\}$. Hence $l(f(q))<\lambda$, and by the induction hypothesis $l(q)<\lambda$. Thus $l(p) \leq \lambda$, and $\sup \left\{l(p): p \in f^{-1}(x)\right\}=\lambda$.

Telgarsky asked $[\mathrm{T}]$ if the image under a closed map of scattered space must be scattered. It is true if the domain has finite length. After constructing the following example this author discovered that the example is contained in $\left[\mathrm{KR}_{1}\right]$ (Proposition 1.11 and Remark 1.12) using the $c$-process, a method of constructing spaces defined in $\left[\mathrm{KR}_{2}\right]$. However, the description here is quite simple, the 
construction using only standard concepts. It is also clear from the description below that the domain and range are suborderable, a fact neither mentioned nor apparent in $\left[K R_{1}\right]$.

Example 2. There is a closed onto map with discrete fibers $f: P \rightarrow X$ where $P$ and $X$ are suborderable (orderable with a more complex construction), $P$ is scattered, $l(P)=\omega_{0}$, and $X$ is perfect.

Let $X_{0}$ be $\omega_{1}+1-\{\alpha: \alpha$ a countable limit ordinal $\}$ with an admissible order under which 0 is its first and $\omega_{1}$ its last point. For all nonzero $n \in \omega_{0}+1$ given the lexicographic product $X_{0}^{n}$, let $X_{n}=\left\{\left\langle x_{i}\right\rangle_{i<n} \in X_{0}^{n}\right.$ : there exist $i_{0}<n$ such that $x_{i_{0}}=\omega_{1}, x_{i} \neq \omega_{1}$ for all $i<i_{0}$, and $x_{i}=0$ for all $\left.i>i_{0}\right\}$. Let $P=\bigoplus\left\{X_{n}: 0<n<\omega_{0}\right\}, X=X_{\omega_{0}}$, and for $0<n<\omega_{0}$ define $f\left(\left\langle x_{i}\right\rangle_{i<n}\right)=$ $\left\langle x_{i}\right\rangle_{i<\omega_{0}}$, where $x_{i}=0$ for all $i \geq n$ and $\left\langle x_{i}\right\rangle_{i<n} \in X_{n}$.

Informally for each nonzero $n<\omega_{0}, X_{n+1}$ is obtained from $X_{n}$ by attaching to each isolated $p \in X_{n}$ an $\omega_{1}$ type sequence of isolated points converging to $p$. $X$ is the limit of this process.

Noting that each countable subset of $X$ is closed, it is routing to show this construction works.

The range can be made orderable by letting $X_{1}$ be a singelton and for each nonzero $n<\omega_{0}$, obtaining $X_{n+1}$ from $X_{n}$ by attaching to each isolated $p \in$ $X_{n}$ an $\omega_{1}$ type sequence and an $\omega_{1}^{*}$ type sequence of isolated points both converging to $p$.

\section{Conclusions}

As implied in the introduction, Theorems 1 and 2 are simplified if the space is paracompact. In particular conditions (1) and (3)(a) are satisfied. Nothing weaker than paracompactness can eliminate condition (3).

If the space is first countable, condition (2) and the interlacing property at each point are satisfied. (Note $\omega_{1} \times\left(\omega_{0}+1\right)$ is scattered, first countable, and strongly collectionwise Hausdorff. But it is not orderable.)

I. Juhász asked if some condition weaker than compactness simplifies the interlacing property. By Lemma 3 if $X$ is countably compact, then the interlacing property is that of $\left[\mathrm{P}_{1}\right]$, namely no point $p$ is the limit of two disjoint sequences closed in $X-\{p\}$, one of which has uncountable cardinality. If $X$ is Lindelöf, for the interlacing property we have no point $p$ is the limit of two disjoint sequences closed in $X-\{p\}$, one of which has cardinality greater than $\omega_{1}$ or is homeomorphic to a stationary subset of $\omega_{1}$. For $X \alpha$-Lindelöf, $\alpha>\omega_{0}$, there are analogous ordinal restrictions on the interlacing property.

Note for the interlacing property these conditions need only be local. Moreover by [T] if a paracompact scattered space $X$ satisfies a condition locally, then $X$ is a disjoint union of clopen sets each satisfying the condition.

Question 1. For an ordered space $X$ is $X^{*}$ (described in Lemma 2) the union of $X$ and the set of its far points in $v X$ ? 
Question 2. Is there a "nice" necessary condition for Theorem 1 implying conditions (2) and (3)?

A $T_{1}$ space $X$ is monotonically normal [HeaLZ] if to each pair $\langle U, x\rangle$ with $U$ open $\subset X$ and $x \in U$, there is an open set $U_{x}$ such that (i) $x \in U_{x} \subset U$, and (ii) if $U_{x} \cap V_{y} \neq \varnothing$, then $x \in V$ or $y \in U$.

Every orderable space is monotonically normal. However the next example shows that monotone normality is not the nice condition sought above. This example also illustrates that condition (1) of the ordering property is needed in the theorem as stated.

Example. Consider the cartesian product $\omega_{1} \times\left(\omega_{0}+1\right)$. Let

$$
Y=\left(\omega_{1} \times\left(\omega_{0}+1\right)\right) \cup\{p\}
$$

be its one point compactification. Let $X=Y-\{\langle\alpha, n\rangle: \alpha$ a limit ordinal, $\left.n \in \omega_{0}\right\}$.

$X$ satisfies conditions (1) and (3) of Theorem 1 (in fact $X$ is paracompact), and $p$ has a neighborhood base satisfying all but condition (1) of the ordering property. (All other points satisfy condition (2) of the theorem.)

Although $\omega_{1} \times\left(\omega_{0}+1\right)$ is not monotonically normal, $X$ is. To see this first note $\left(\omega_{1} \times\left\{\omega_{0}\right\}\right) \cup\{p\}$ is homeomorphic to $\omega_{1}+1$. So for each pair $\langle q, U\rangle$ with $U$ relatively open $\subset\left(\omega_{1} \times\left\{\omega_{0}\right\}\right) \cup\{p\}$ and $q \in U$, let $U_{q}$ satisfy the conditions given in the definition of monotone normality.

So let $V$ open $\subset X$ and $x \in V$. We define $V_{x}$.

For $x=\langle\alpha, n\rangle$ where $n \in \omega_{0}, x$ is isolated. So define $V_{x}=\{x\}$.

For $x=\left\langle\alpha, \omega_{0}\right\rangle$, there exists $\xi \leq \alpha$ and $n<\omega_{0}$ such that $([\xi, \alpha] \times$ $\left.\left[n, \omega_{0}\right]\right) \cap X$ is open in $X$ and contained in $V$. Let $V_{x}=\left((V \cap[\xi, \alpha])_{x} \times\right.$ $\left.\left[n, \omega_{0}\right]\right) \cap X . V_{p}$ is defined analogously (where now $n=0$ ).

Question 3. In Theorems 1 and 2 can the length restrictions in condition (3)(b) be eliminated?

Question 4. For Theorem 1 is it possible that condition (1) of the ordering property can be replaced by the following weaker condition? Let $\mathscr{B}=$ $\left\{\bigcap_{\alpha<\beta} I_{\alpha}-I_{\beta}: 0<\beta<\tau\right\}$. Then for each $p \in X$ there is a neighborhood base of $p$ each member of which contains every member of $\{B: B \in \mathscr{B}$ and $p \notin B\}$ which it intersects. (So for $B^{\prime} \subseteq \mathscr{B}$ and any two sets $C$ and $D$ each consisting of one point from each member of $\mathscr{B}^{\prime}, C$ and $D$ have the same accumulation points.)

Using the argument in $\left[\mathrm{P}_{1}\right]$ with the type of modifications used in the proof of Theorem 1 in this paper, we prove the analogue of Theorem 2 in $\left[\mathrm{P}_{1}\right]$, where in place of condition (2) of that result we have the following. There is no subset $Y$ of $X$ such that $Y=\bigcup_{s \in S} X_{s}$ where: the $X_{s}$ 's are pairwise disjoint; $S$ is a stationary subset of some ordinal; for each $s \in S, X_{s}=A_{s} \cup\left\{p_{s}\right\} \cup B_{s}$, where $A_{s}$ and $B_{s}$ are disjoint sequences closed in $Y-\left\{p_{s}\right\}$ and converging to $p_{s}$, and either $A_{s}$ and $B_{s}$ have the same cardinality or $B_{s}$ is homeomorphic to a stationary set, and a basic neighborhood of $p_{s}$ is the union of a basic 
neighborhood of $p_{s}$ in $X_{s}$ with $\bigcup\left\{X_{\xi}: \alpha<\xi<s\right.$, and $\left.\xi \in S\right\}$ for some $\alpha<s ;$ and $\left\{p_{s}\right\}_{s \in S}$ is homeomorphic to $S$.

(See Example 2.1 minus the point $p$ in $\left[\mathrm{P}_{1}\right]$. Letting $\tau_{2}>\omega_{0}$ corrects a misprint there.)

\section{REFERENCES}

[B] J. W. Baker, Compact scattered spaces homeomorphic to a ray of ordinals, Fund. Math. 76 (1972), 19-27.

[E] R. Engelking, General topology, Polish Scientific Publishers, Warsaw, 1977.

[EL] R. Engelking and D. J. Lutzer, Paracompactness in ordered spaces, Fund. Math. 94 (1977), 49-58.

[F] V. V. Fedorčuk, On ordered spaces, Dokl. Akad. Nauk SSSR 169 (1966), 777-780; English transl., Soviet Math. Dokl. 7 (1966), 1011-1014.

[GHen] L. Gillman and M. Henriksen, Concerning rings of continuous functions, Trans. Amer. Math. Soc. 77 (1954), 340-362.

[HeaLZ] R. W. Heath, D. J. Lutzer, and P. L. Zenor, Monotonically normal spaces, Trans. Amer. Math. Soc. 178 (1973), 481-494.

[KR $]$ V: Kannan and M. Rajagopalan, Scattered spaces. II, Illinois J. Math. 21 (1977), 735-751. $\left[\mathrm{KR}_{2}\right]$ - Constructions and applications of rigid spaces. I, Adv. in Math. 29 (1978), 89-129.

[M] G. Moran, Order-two continuous Hausdorff images of compact ordinals, Israel J. Math. 42 (1982), 75-86.

$\left[\mathrm{P}_{1}\right] \quad$ S. Purisch, The orderability of compact scattered spaces $(\mathrm{H} . \mathrm{R}$. Bennett and D. J. Lutzer, eds.), Topology and Order Structure, Part II, Tract 169, Math. Centre Tracts, Amsterdam, 1983, pp. 53-61.

$\left[\mathrm{P}_{2}\right] \quad-$ Scattered compactifications and the orderability of scattered spaces. II, Proc. Amer. Math. Soc. 95 (1985), 636-640.

[R] M. E. Rudin, Lectures on set theoretic topology, CBMS Regional Conference Series in Math., no. 23, Amer. Math. Soc., Providence, R.I., 1975, reprinted 1977, Problems, p. 62.

[S] V. Slucká, personal communication.

[T] R. Telgarsky, Total paracompactness and paracompact dispersed spaces, Bull. Acad. Polon. Sér. Sci. Math. Astronom. Phys. 16 (1968), 567-572.

Department of Mathematics, Tennessee Technological University, Cookeville, TenNESSEE 38505 